\title{
Perspectivas del Uso de la Amalgama Dental bajo el Convenio de Minamata: Tendencias Nacionales e Internacionales
}

\author{
Perspectives of the Use of Dental Amalgam Under the Minamata Convention: National \\ and International Tendencies
}

Perspectivas para o uso de amálgama dental sob a Convenção de Minamata: tendências nacionais e internacionais

Gabriel Jaime Gómez ${ }^{1 \otimes}$

1. Ingeniero Metalúrgico, Gerencia de Investigación y Desarrollo, New Stetic S.A.

Fecha correspondencia:

Recibido: septiembre de 2019.

Aceptado: febrero de 2020.

Forma de citar:

Gomez GJ. Perspectivas del Uso de la Amalgama Dental bajo el Convenio de Minamata: Tendencias Nacionales e Internacionales. Rev. CES Odont 2020; 33(1): 53-63.

\section{Open access}

(C) Derecho de autor

Licencia creative commons

Ética de publicaciones

Revisión por pares

Gestión por Open Journal System DOl: http://dx.doi.org/10.21615/ cesodon.33.1.7 ISSN 0120-971X e-ISSN 2215-9185

\section{Resumen}

El objetivo de este artículo de revisión es abordar el tema de la amalgama dental en relación con el Convenido de Minamata Sobre el Mercurio. Las decisiones que, al amparo de dicho convenio, se vienen tomando en el mundo, incluyendo Colombia, y que afectan la continuidad de la amalgama como material de restauración dental, siendo esto agravado por los altos niveles de prevalencia de la caries dental a nivel global. Se revisa la posición de algunas organizaciones internacionales y sus decisiones basadas en lo que la evidencia científica disponible muestra sobre la toxicidad y biocompatibilidad de la amalgama, y sus posibles materiales de reemplazo. Se revisa el panorama nacional y el impacto de las decisiones gubernamentales sobre la profesión odontológica.

Palabras clave: Amalgama dental, mercurio, Convenio de Minamata, biocompatibilidad, caries dental.

\section{Abstract}

The aim of this review article is to address the issue of dental amalgam in relation to the Minamata Convention on Mercury. The decisions that, under this convention, are being taken in the world, including Colombia, and which affect the continuity of amalgam as dental restoration material, this is being aggravated due to the high levels of prevalence of dental caries globally. The position of some international organizations, their decisions based on the available scientific evidence about the toxicity and biocompatibility of amalgam, and its possible replacement materials are reviewed. The national landscape and the impact of government decisions on the dental profession are reviewed.

Keywords: Dental amalgam, mercury, Minamata Convention, biocompatibility, dental caries. 


\section{Resumo}

O objetivo deste artigo de revisão é abordar a questão da amálgama dental em relação ao Acordo de Minamata sobre Mercúrio. As decisões que, sob este acordo, estão sendo tomadas no mundo, incluindo a Colômbia, e que afetam a continuidade do amálgama como material de restauração dentária, agravadas pelos altos níveis de prevalência mundial de cárie dentária. A posição de algumas organizações internacionais e suas decisões são revisadas com base no que as evidências científicas disponíveis mostram sobre a toxicidade e biocompatibilidade do amálgama e seus possíveis materiais de substituição. A paisagem nacional e o impacto das decisões do governo na profissão odontológica são revistos.

Palavras-chave: Amálgama dentária, mercúrio, Convenção de Minamata, biocompatibilidade, cárie dentária.

\section{Introducción}

La amalgama hace parte de la familia de biomateriales destinados a la restauración directa de dientes posteriores. Históricamente, la amalgama ha sido, hasta ahora, la alternativa más utilizada por los odontólogos gracias a características como su costo/ beneficio, altas propiedades mecánicas, comprobada longevidad en boca y uso recomendado en personas con pobres hábitos de higiene bucal y alto riesgo de sufrir caries.

Defendida por muchos, pero también con muchos detractores, la amalgama ha sido objeto de múltiples estudios asociados con su biocompatibilidad y al hecho de que se le pueda llamar biomaterial cuando en su composición está el mercurio, un metal reconocido por su toxicidad para con los seres vivos y el medio ambiente.

En este artículo se hace una revisión general sobre la posición que actualmente tienen algunos organismos internacionales, incluyendo Colombia, con respecto a la vigencia de la amalgama como material de restauración dental. También se revisa cómo el uso de la amalgama se ve inevitablemente afectado por la tendencia mundial en la disminución gradual del uso industrial del mercurio, enmarcado en lo que se conoce como el Convenio de Minamata. Para esto se consultaron los sitios web y varias publicaciones de organizaciones como la American Dental Association (ADA), the FDI World Dental Federation, the Canadian Dental Association (CDA), the British Dental Association (BDA), the Council of European Dentists, entre otras fuentes.

También se trae a colación un panorama general de la situación de la caries dental en el mundo, pues la presencia y el tratamiento de esta enfermedad bucodental tiene relación directa con las alternativas de restauración actualmente disponibles.

\section{El Convenio de Minamata y la amalgama}

El Convenio de Minamata es el primer acuerdo ambiental mundial negociado en el siglo XXI. El texto fue adoptado el 10 de octubre de 2013, abierto a su firma hasta el 9 de octubre de 2014 y en agosto de 2017 entró en vigor.

En relación con la amalgama, el Convenio de Minamata establece que las medidas que han de adoptarse para reducir su uso tendrán en cuenta las circunstancias nacionales de cada país y las orientaciones internacionales pertinentes, incluyendo medidas para la prevención de la caries, la promoción de la salud, investigación, desarrollo y uso de materiales de calidad sin mercurio, educación y capacitación a estudiantes y odontólogos sobre el uso de alternativas sin mercurio y promoción de las mejores 
prácticas, desincentivar el uso de la amalgama, uso de la amalgama en forma encapsulada, y promoción de las mejores prácticas ambientales en los consultorios para reducir las liberaciones de mercurio al ambiente (1).

\section{Aspectos ambientales, de toxicidad y biocompatibilidad de la amalgama}

Es claro que todas las personas están expuestas a cierto nivel de mercurio, como lo afirma la Organización Mundial de la Salud (2), situación que es agudizada por las fuentes antropogénicas de mercurio (3).

Si bien la minería artesanal y a pequeña escala del oro como principal fuente antropogénica de mercurio es de particular interés en el radar mundial, los productos con mercurio añadido, dentro de los cuales está la amalgama, también son abordados dentro de las estrategias tendientes a disminuir gradualmente su producción y utilización. Particularmente la industria productora de aleaciones para amalgama representa entre un 6 y $10 \%$ del consumo mundial de mercurio, y de ese consumo total de mercurio para uso odontológico, se estima que el 25\% termina en los vertederos junto con los desechos sólidos. Adicionalmente, se calcula que su contribución a las emisiones de mercurio es menor al $1 \%$ en lo relacionado con la cremación de restos humanos, por ejemplo (3).

Sin embargo, desde el punto de vista ambiental, la amalgama no es la principal fuente de contaminación por mercurio (4). Afortunadamente los residuos de amalgama son completamente reciclables, pudiendo recuperar el mercurio con el fin de reincorporarlo a la cadena productiva. Adicionalmente, con el fin de disminuir el impacto de los residuos generados por su uso, hay que tomar conciencia sobre la importancia que tiene no extraer desde los pacientes las restauraciones clínicamente funcionales simplemente por conceptos estéticos, ya que esto no sólo aumenta la generación de residuos, sino que expone al profesional y al paciente a vapores de mercurio, además de que este último sacrificará una restauración funcional por otro material que, en un menor tiempo, posiblemente requiera de un nuevo reemplazo.

Y es que, desde el punto de vista del desempeño clínico, biocompatibilidad y toxicidad para el paciente, la evidencia disponible no sólo muestra tasas de supervivencia mayor para las restauraciones de amalgama $(5,6)$, sino que las liberaciones de mercurio desde éstas son tan bajas que no alcanzan a llegar a niveles tóxicos y, por ende, no hay evidencia concluyente sobre claras afectaciones a nivel sistémico $(7,8)$.

\section{Posiciones internacionales}

En los reportes de "What Others Say" sobre la amalgama dental publicados por la ADA en marzo de 2013 y abril de 2016, esta asociación cita la posición de diversas organizaciones con respecto a la seguridad y efectividad de la amalgama. A continuación algunas citaciones.

Alzheimer's Association: De acuerdo con la mejor evidencia científica disponible, no existe una relación entre las restauraciones de amalgama y el Alzheimer. Muchos científicos consideran que estos estudios son evidencia convincente de que la amalgama dental no es un factor de riesgo importante para dicha enfermedad (9). Las agencias de salud pública, incluida la Food and Drug Administration, el Servicio de Salud Pública de los Estados Unidos y la Organización Mundial de la Salud, respaldan el uso de la amalgama como material seguro, resistente y de bajo costo (10). 
American Academy of Pediatrics: Aunque las amalgamas son una fuente de exposición al mercurio y están asociadas con una excreción ligeramente más alta de mercurio en la orina, no hay evidencia científica de efectos tóxicos clínicos medibles aparte de reacciones de hipersensibilidad poco frecuentes. Un panel de expertos de los National Institutes of Health ha concluido que la evidencia existente indica que las amalgamas no representan un riesgo para la salud y no deberían ser reemplazadas simplemente para disminuir la exposición al mercurio (9).

National Multiple Sclerosis Society: No hay evidencia científica que relacione el desarrollo o empeoramiento de la esclerosis múltiple (EM) con restauraciones de amalgama y, por lo tanto, no hay motivo para que se eliminen esas restauraciones. A través de los años se ha afirmado que el mercurio que se escapa de las restauraciones de amalgama daña el sistema inmune y causa una amplia gama de enfermedades, sin embargo, no hay evidencia científica de que la intoxicación por metales pesados sea responsable del inicio o el empeoramiento de la EM. No hay ninguna razón para que se retire o sustituyan las amalgamas. Este es un procedimiento muy costoso sin beneficio comprobado para personas con EM (9).

The National Institute of Dental and Craniofacial Research (NIDCR): Dos estudios, uno en Europa y otro en Estados Unidos, llegaron a la conclusión, de forma independiente, que los niños cuyas caries estaban tratadas con amalgamas no tenían efectos adversos en su salud. Los hallazgos no incluyeron pérdida detectable de inteligencia, memoria, coordinación, concentración, conducción nerviosa o función renal durante los 5 a 7 años que los niños fueron evaluados $(9,10)$.

Environmental Protection Agency (EPA): Desde la década de 1990, varias agencias federales han revisado la literatura científica en busca de vínculos entre la amalgama y los problemas de salud. Según los Centers for Disease Control and Prevention (CDC), hay poca evidencia científica de que la salud de la gran mayoría de las personas con amalgamas esté comprometida, ni que la eliminación de las restauraciones de amalgama tenga un efecto beneficioso en la salud (9).

Food and Drug Administration: La FDA ha revisado la mejor evidencia científica disponible para determinar si los bajos niveles de vapor de mercurio asociados con las restauraciones de amalgama son motivo de preocupación. La FDA considera que las restauraciones de amalgama son seguras para adultos y niños de 6 años en adelante. La cantidad de mercurio medida en el cuerpo de las personas con amalgamas está muy por debajo de los niveles asociados con efectos adversos para la salud. La FDA concluye que los datos existentes respaldan el hallazgo de que los bebés no están en riesgo de efectos adversos para la salud debido a leche materna de mujeres expuestas al vapor de mercurio de la amalgama (9). Se dispone de datos clínicos muy limitados o inexistentes sobre los resultados de salud a largo plazo en mujeres embarazadas y sus fetos en desarrollo, y en niños menores de seis años, incluidos los bebés que son amamantados. Sin embargo, la cantidad estimada de mercurio en la leche materna atribuible a la amalgama es baja y está muy por debajo de los niveles generales de ingesta oral que la EPA considera segura. Algunas personas tienen alergia o sensibilidad al mercurio u otros componentes de la amalgama, pudiendo desarrollar lesiones orales u otras reacciones de contacto. En tal caso, se puede discutir otras opciones de tratamiento con el odontólogo (10). 
Por su parte, la FDI afirma que, pese a los grandes avances conseguidos en su prevención, la caries dental sigue siendo una de las enfermedades más comunes en todo el mundo y que, de entre todos los materiales que se utilizan para la restauración directa, la amalgama es uno de los que ofrecen mayor durabilidad. Adicionalmente, las amalgamas se utilizan ampliamente para la restauración de dientes debido a su fácil uso, reducido coste y propiedades mecánicas y bacteriostáticas. Al día de hoy la amalgama lleva más de 150 años en uso y a pesar de los enormes esfuerzos de investigación invertidos en el desarrollo de materiales alternativos, hasta la fecha no se ha podido encontrar un sustituto universal que permita reemplazarlas. Por ese motivo, los odontólogos deben poder disponer de la amalgama como opción terapéutica. De hecho, La FDI reiteró la principal conclusión de la Declaración de Consenso de la OMS sobre la amalgama aprobada por la Asamblea General de la FDI en 1997, que afirma que el peso de la evidencia actual sugiere que los materiales de restauración dental, incluidas las amalgamas, son seguros y efectivos. Obviamente, para llegar a la desaparición de las amalgamas habrá que reducir el uso de las mismas adoptando medidas orientadas a crear conciencia pública sobre la importancia de la salud bucodental, fomentar la cooperación entre los diferentes actores, enfatizar sobre la importancia de la evaluación de las necesidades particulares de cada paciente, prevención de caries, promoción de la salud, garantizar buenas prácticas en el manejo del mercurio, la amalgama y su disposición final, y mejoramiento y desarrollo de materiales de alta calidad (11).

\section{Otras posiciones}

Council of European Dentists: la amalgama debe seguir siendo parte de las alternativas que tiene el odontólogo para satisfacer las necesidades de los pacientes, a los cuales, por supuesto, no se les debe negar la libertad de elección con respecto al tratamiento. Como alternativa, la amalgama continúa siendo un material apropiado para muchas restauraciones debido a su facilidad de uso, durabilidad y rentabilidad, y su eliminación gradual debe ir acompañada del desarrollo de un material de restauración alternativo adecuado (12).

\section{CDA}

La evidencia científica actual sobre el uso de la amalgama respalda que ésta es un material de restauración eficaz y seguro que proporciona una solución duradera para una amplia gama de situaciones clínicas, además de que ofrece beneficios sobre otros materiales con respecto a la facilidad de uso y la longevidad, especialmente en pacientes con un alto riesgo de caries dental. Se considera innecesario y poco aconsejable reemplazar obturaciones de amalgamas funcionales. Las circunstancias y los hechos clínicos específicos deben ser discutidos por los odontólogos con sus pacientes, con el fin de seleccionar el material más apropiado para la situación clínica en particular. Los pacientes que solicitan el reemplazo de restauraciones de amalgama deben recibir suficiente información para comprender las implicaciones de su decisión (13).

\section{$B D A$}

El uso de la amalgama se debe a que es un material comprobado, de fácil manipulación, duradero, resistente, bacteriostático, rentable y sencillo y rápido de colocar en cavidades de todos los tamaños. La amalgama sigue siendo uno de los materiales de restauración disponibles para los odontólogos, que les permite brindar un tratamiento apropiado para el paciente. La amalgama ha estado en uso y se ha estudiado ampliamente durante más de 150 años, donde su seguridad y durabilidad están bien establecidas, y sigue siendo el material más apropiado para una variedad de situa- 
ciones clínicas. No hay justificación para eliminar las restauraciones de amalgama clínicamente satisfactorias como medida de precaución, excepto en los pacientes diagnosticados con reacciones alérgicas a sus componentes, situación que es de rara ocurrencia (14).

International Association for Dental Research (IADR): Con base en la mejor evidencia disponible, la IADR confirma la seguridad de la amalgama para la población en general que no presenta alergia a sus componentes o enfermedades renales graves. La IADR apoya mantener su disponibilidad como la mejor opción de restauración cuando las alternativas son menos óptimas por razones clínicas, económicas o prácticas. La IADR apoya la estrategia de reducción gradual descrita en el Convenio de Minamata, pero enfatiza en la necesidad de aumentar los esfuerzos de prevención de enfermedades orales para reducir la necesidad de cualquier tipo de material restaurador, y en una mayor investigación sobre nuevos materiales restauradores biocompatibles y amigables con el medio ambiente, que tengan una longevidad clínica a largo plazo igual o mejorada, y rentabilidad en comparación con las restauraciones de amalgama (15).

Es interesante como en Europa, por ejemplo, las medidas adoptadas alrededor del uso de la amalgama y consignadas en Reglamento (UE) 2017/852 del Parlamento Europeo y del Consejo del 17 de mayo de 2017 sobre el mercurio, contempla, entre varias medidas, sólo usar cápsulas predosificadas, separadores de amalgama en los consultorios y el posible uso de la amalgama hasta el 2030 (16).

A nivel mundial ya varios países tienen un camino recorrido en relación con la reducción gradual de la amalgama, países como Suiza, Suecia, Noruega, Dinamarca, Finlandia, Países Bajos, Japón, Hungría y Singapur, son un ejemplo de esto (17). Adicionalmente, en la Lista de las Partes publicada en la página web del Convenio de Minamata (mercuryconvention.org), se reportan 30 países por África, 26 por Asia, 13 por Europa Central y del Este, 23 por América Latina y el Caribe, y 21 por Europa Occidental y otros grupos, que se han adherido, ratificado, aceptado o aprobado dicho convenio, donde cada país establece sus estrategias de reducción gradual de la amalgama basadas en sus circunstancias particulares.

Pero no se puede olvidar que la caries dental es la enfermedad crónica más extendida en el mundo. Es la enfermedad más frecuente en la infancia pero afecta a todas las edades a lo largo de la vida. Los datos actuales muestran que la caries no tratada en dientes permanentes tiene una prevalencia global (promediando todas las edades) del $40 \%$. Incluso en los países con alto nivel de ingresos, más de la mitad de las caries permanecen sin tratamiento. Al igual que para otras enfermedades bucodentales, la caries comparte los mismos determinantes sociales y las mismas desigualdades en salud (18).

\section{Panorama colombiano}

En Colombia la normatividad sobre el mercurio comenzó con la Ley 1658 de julio de 2013, con el fin de fijar disposiciones para su comercialización, uso, reducción y eliminación a nivel nacional. Luego, en octubre del mismo año, el País fue firmante del Convenio de Minamata, el cual fue aprobado mediante la Ley 1892 de mayo de 2018 y ratificado en agosto de 2019. A esto se suma lo establecido en el Decreto 1041 de junio de 2018, a través del cual se modificaron los cupos y fechas límites de importación y uso del mercurio. A la luz de este panorama, en Colombia se podrá importar y utilizar mercurio para la fabricación de aleaciones para amalgama hasta julio de 2023, aunque su uso clínico no está restringido después de dicha fecha. Sin 
embargo, el Gobierno Nacional ya está tomando medidas enfocadas a desincentivar el uso de la amalgama mediante políticas para su reducción gradual (19).

Sin embargo, esta situación también hay que verla a la luz de los niveles de prevalencia de caries en el país. En este sentido, las altas prevalencias de caries ya se habían reportado en el IV Estudio Nacional de Salud Bucal -ENSAB IV- realizado en los años 2013-2014. En Colombia, según dicho estudio, la prevalencia de caries a la edad de 5 años era de $52.20 \%$ (dentición temporal), a los 12 años era de $37.45 \%$ (dentición permanente) y a los 18 años era de $47.79 \%$, niveles que podían llegar a prevalencias de $82.63 \%$ a los 5 años y $93.80 \%$ a los 18 años si se consideraban otros aspectos adicionales (20).

Las bajas coberturas en procedimientos educativos y de cuidado preventivo, y el limitado acceso a los servicios de consulta odontológica para la atención temprana y resolutiva, se evidencian en la presencia de alteraciones bucales a lo largo de la vida, como es el caso de la caries dental y sus secuelas como la pérdida dental. Es así como, en las edades adultas, la población entre 20 y 34 años ya presenta un $88 \%$ de experiencia de caries y un $52,81 \%$ se encuentra sin atención (21).

La profesión odontológica nacional se enfrenta entonces a una situación complicada en relación con la desaparición gradual de la amalgama. La situación de un país con altos índices de caries donde:

- No se cuenta con materiales sustitutos que ofrezcan mejores, o por lo menos iguales, niveles de desempeño clínico a mediano y largo plazo, lo que conlleva a sobrecostos para el sistema de salud, no sólo por el precio más alto en sí de los materiales alternativos en relación con la amalgama, sino porque por lo general dichos materiales alternativos presentan una mayor tasa de fracasos, lo que se traduce en un mayor número de reemplazos en el tiempo, terminando en algunos casos en tratamientos endodónticos.

- Una evidente disminución de la enseñanza y uso de la amalgama en las facultades de odontología.

- La avalancha de exigencias estéticas donde muchas veces la selección del material restaurador no obedece a la necesidad y condiciones clínicas particulares del paciente, es decir, se coloca la estética por encima de la función.

Con este panorama, Colombia se enfrenta a una decisión que, en las circunstancias actuales, afectará de forma negativa en el mediano y largo plazo la salud dental de la población nacional.

\section{Amalgama y materiales alternativos}

Como material de restauración dental, la amalgama aún no cuenta con un verdadero sustituto (4), ya que los demás materiales de restauración disponibles son alternativos, por lo que estos últimos no necesariamente son ideales para cualquier tipo de situación clínica (22). Tampoco puede afirmarse que, por contener mercurio, la amalgama en sí misma sea un material tóxico y no deba considerarse biocompatible. La amalgama no es mercurio (4), y no es el único material de restauración dental con el potencial de generar algún tipo de reacción adversa en el personal dental y en los pacientes. Se reporta que dada la genotoxicidad demostrada de algunos monómeros liberados mientras se colocan las resinas, el personal dental debe tomar 
medidas de control de exposición adecuadas al emplear estos materiales (23). Los profesionales pueden estar expuestos a estos monómeros ya sea por inhalación o por contacto directo con la piel, produciendo reacciones dermatológicas (24).

En relación con los pacientes, la liberación de monómeros desde las resinas se debe, principalmente, a una polimerización incompleta y a los procesos de degradación en el entorno oral (23), pudiendo producir reacciones cutáneas o de las mucosas en los pacientes, incluyendo reacciones anafilactoides o liquenoides, y otras respuestas alérgicas (24). Los estudios in vitro han demostrado la genotoxicidad del TEGDM, MMA, HEMA y sus metabolitos (23).

Las reacciones alérgicas producidas tanto por las amalgamas como por las resinas de fotocurado, son de rara ocurrencia (25). Cada paciente deberá discutir con su odontólogo la mejor alternativa de tratamiento de acuerdo con su condición clínica particular (22).

La realidad es que la amalgama sigue mostrando, gracias a sus propiedades, un desempeño clínico superior en relación con su longevidad a mediano y largo plazo, evidenciándose esto en menores tasas de fracaso en comparación con otros materiales de restauración directa.

\section{Conclusiones}

Son bien conocidos los efectos tóxicos que tiene el mercurio en el ambiente y la salud de las personas, sin embargo, esto en sí mismo no convierte a la amalgama en un material tóxico, ya que el mercurio se encuentra formando una aleación con los otros metales presentes, confiriéndole características físicas y químicas muy diferentes a las que posee en su estado elemental. Caso contrario son otras fuentes de exposición como el pescado contaminado, fuente de metil mercurio, la forma química más tóxica del mercurio.

La presencia de mercurio en cualquier actividad laboral requiere de prácticas adecuadas de seguridad y salud en el trabajo, orientadas a proteger la salud de las personas laboralmente expuestas. En el ámbito odontológico esto significa utilizar, preferiblemente, aleación para amalgama en presentaciones comerciales predosificadas, y garantizar el almacenamiento y disposición final adecuados de los residuos mercuriales. Con respecto a esto último, los residuos de amalgama son perfectamente reciclables y aprovechables.

Pese a la controversia, la amalgama se ratifica hoy como un biomaterial efectivo y seguro para una amplia variedad de situaciones clínicas. Su elección y uso como material de restauración dental debe tener como sustento el conocimiento de los materiales y una técnica correcta de parte del profesional, y la evaluación de la necesidad particular del paciente. Sería injusto para los pacientes no ofrecerles la posibilidad de uso de amalgama en los casos en donde estuviera indicada. También hay que reflexionar en el cambio de restauraciones de amalgama funcionales por otros materiales alternativos, simplemente por conceptos estéticos y con el desconocimiento por parte del paciente en cuanto a las posibles consecuencias de su decisión.

La implementación del Convenio de Minamata plantea hoy grandes retos alrededor del uso de la amalgama. Es evidente que la desaparición gradual de la amalgama del abanico de materiales restauradores, no se debe a que se haya comprobado categóricamente sus efectos tóxicos sobre la población en general. Más bien, la tendencia 
actual por los materiales estéticos y la preocupación ambiental por los residuos mercuriales es lo que lleva a su desuso gradual. Esto hace pertinente dirigir la mirada hacia la investigación para el mejoramiento de los materiales alternativos actuales y el desarrollo de nuevos materiales de restauración de alto desempeño. Tampoco se puede olvidar la necesidad de fortalecer los programas de promoción de la salud y de prevención de la caries, y la necesidad de educar más para restaurar menos, independiente del material.

La eliminación gradual de la amalgama debe obedecer a estrategias donde estén involucrados el Estado, los profesionales de la salud, la industria dental y la academia, de tal manera que las decisiones propendan por el bienestar de la población, especialmente para los sistemas y programas de salud que atienden población vulnerable, rural o de difícil acceso (26), y no simplemente por intereses económicos. Adicionalmente es importante considerar el sobre costo que implica para los sistemas y programas de salud reemplazar totalmente la amalgama por materiales más costosos y con una expectativa de vida en boca más corta, y cuando es el paciente el que debe pagar por el tratamiento de restauración, como mínimo tiene derecho a ser informado imparcialmente por parte de su odontólogo con el fin de que tome una decisión basada en conocimiento.

\section{Referencias}

1. Naciones Unidas. Convenio de Minamata Sobre el Mercurio. Texto y Anexos [Internet]. Septiembre de 2017. [Citado 2020 feb. 11]. Disponible en: http://mercuryconvention.org/Portals/11/documents/Booklets/COP1\%20version/Minamata-Convention-booklet-sp-full.pdf

2. Organización Mundial de la Salud. El Mercurio y la Salud [Internet]. 31 de marzo de 2017. [Citado 2020 feb. 11]. Disponible en: http://www.who.int/es/news-room/ fact-sheets/detail/mercury-and-health.

3. Centro Coordinador Convenio Basilea - Centro Regional Convenio de Estocolmo para América Latina y el Caribe. Informe. El Convenio de Minamata Sobre el Mercurio y su Implementación en la Región de América Latina y el Caribe [Internet]. Abril de 2014. [Citado 2020 feb. 11]. Disponible en: http://www.mercuryconvention.org/ Portals/11/documents/publications/informe Minamata LAC ES FINAL.pdf

4. Kelly R. Position Statement: Dental Amalgam [Internet]. American College of Prosthodontists. November 4, 2014. [Citado 2020 feb. 11]. Disponible en: https:// www.prosthodontics.org/about-acp/position-statement-dental-amalgam/

5. Bernardo M, Luis H, Martin M, Leroux B, Rue T, Leitão J et al. Survival and reasons for failure of amalgam versus composite posterior restorations placed in a randomized clinical trial. JADA 2007;138(6):775-83.

6. Kopperud SE, Tveit AB, Gaarden T, Sandvik L, Espelid I. Longevity of posterior dental restorations and reasons for failure. Eur J Oral Sci 2012; 120(6): 539-548.

7. Canadian Agency for Drugs and Technologies in Health. Composite Resin Filling Materials: A Review of Safety, Cost-Effectiveness and Guidelines - An Update [Internet]. 15 October 2015. [Citado 2020 feb. 11]. Disponible en: https://www.cadth. ca/sites/default/files/pdf/htis/oct-2015/RC0711\%20Composite\%20Resin\%20 Safety\%20Final.pdf 
8. Canadian Agency for Drugs and Technologies in Health. IN BRIEF A Summary of the Evidence Composite Resin Versus Amalgam for Dental Restorations [Internet]. March 2018. [Citado 2020 feb. 11]. Disponible en: https://cadth.ca/sites/ default/files/pdf/ht0021 in brief.pdf

9. American Dental Association. "What Others Say" About Dental Amalgam. Last Updated: March 27, 2013.

10. American Dental Association. Dental Amalgam: What Others Say [Internet]. March 2019. [Citado 2020 feb. 11]. Disponible en: http://www.ada.org/en/pressroom/press-kits/dental-fillings-press-kit/dental-amalgam-what-others-say

11. FDI World Dental Federation. Las Amalgamas Dentales y el Convenio de Minamata Sobre el Mercurio [Internet]. Septiembre de 2014. [Citado 2020 feb. 11]. Disponible en: https://www.fdiworlddental.org/sites/default/files/media/documents/6-fdi draft ps-dental amalgam and minamata final es.pdf

12. Council of European Dentists. CED Resolution. Dental Amalgam: 2013 Update [Internet]. 22 November 2013. [Citado 2020 feb. 11]. Disponible en: https://www. omd.pt/content/uploads/2019/08/201311-ced-amalgam-update.pdf

13. Canadian Dental Association. CDA Position on Dental Amalgam [Internet]. March 2014. [Citado 2020 feb. 11]. Disponible en: https://www.cda-adc.ca/en/about/ position statements/amalgam/

14. British Dental Association. Amalgam. May 2017.

15. International Association for Dental Research. Safety of Dental Amalgam [Internet]. June 2019. [Citado 2020 feb. 11]. Disponible en: http://www.iadr.org//ADR/ About-Us/Policy-Statements/IADR-Science-Policy/Safety-of-Dental-Amalgam.

16. Parlamento Europeo y Consejo de la Unión Europea. Reglamento (UE) 2017/852 Sobre el Mercurio [Internet]. 17 de mayo de 2017. [Citado 2020 feb. 11]. Disponible en: https://eur-lex.europa.eu/legal-content/ES/TXT/PDF/?uri=CELEX:32017R0852\&from $=F R$

17. UN Environment Program. Lessons from Countries Phasing Down Dental Amalgam Use [Internet]. March 2016. [Citado 2020 feb. 11]. Disponible en: http://archive.zoinet.org/web/sites/default/files/publications/Dental Amalgam spreads.pdf

18. FDI World Dental Federation. El Desafío de las Enfermedades Bucodentales. Una Llamada a la Acción Global [Internet]. 2015. [Citado 2020 feb. 11]. Disponible en: https://www.fdiworlddental.org/sites/default/files/media/documents/book spreads oh2 spanish.pdf

19. Ministerio de Salud y Protección Social de Colombia. Lineamiento para el Uso Controlado de la Amalgama Dental, en los Servicios de Odontología. Orientaciones en el Marco de los Compromisos Asumidos en el Convenio de Minamata [Internet]. Marzo 2018. [Citado 2020 feb. 11]. Disponible en: https://www.minsalud.gov.co/sites/rid/Lists/BibliotecaDigital/RIDE/VS/PP/ENT/uso-controlado-amalgama.pdf 
20. Ministerio de Salud y Protección Social de Colombia. Lineamientos Estrategia Incremental de Cuidado y Protección Específica en Salud Bucal para la Primera Infancia, Infancia y Adolescencia "Soy Generación más Sonriente" [Internet]. Marzo de 2019. [Citado 2020 feb. 11]. Disponible en: https://www.minsalud. gov.co/sites/rid/Lists/BibliotecaDigital/RIDE/VS/PP/ENT/lineamientos-soy-generacion-sonriente-2019.pdf

21. Ministerio de Salud y Protección Social de Colombia. Lineamientos Estrategia Incremental de Cuidado Bucal y Protección Específica en Salud Bucal para la Primera Infancia, Infancia y Adolescencia. Estrategia Soy Generación Más Sonriente [Internet]. Febrero de 2018. [Citado 2020 feb. 11]. Disponible en: https://www. medellin.gov.co/irj/go/km/docs/pccdesign/medellin/Temas/Salud O/Programas/Shared \%20Content/Salud\%20Bucal/SOY\%20GENERACION\%20MAS\%20 SONRIENTE\%20V.\%205\%202018.pdf

22. Colgate. Dental Amalgam: A Health Risk? [Internet]. 05/12/2014. [Citado 2020 feb. 11]. Disponible en: https://www.colgate.com/en-us/oral-health/procedures/fillings/dental-amalgam-a-health-risk.

23. Erdal S. Mercury in Dental Amalgam and Resin-Based Alternatives: A Comparative Health Risk Evaluation [Internet]. June 2012. [Citado 2020 feb. 11]. Disponible en: https://www.wfpha.org/tl files/images/Newsletter\%202012/July/ Res\%20Colab\%20Amalgam\%20Risk\%20Final.pdf

24. FDI World Dental Federation. Declaración de Principios de la FDI. Reacciones Negativas a los Materiales a Base de Resinas para Obturaciones Directas [Internet]. Septiembre de 2009. [Citado 2020 feb. 11]. Disponible en: https://www.fdiworlddental.org/sites/default/files/media/documents/Adverse-reactions-to-resin-based-direct-filling-materials-2009-Sp.pdf

25. Ucar Y, Brantley W. Biocompatibility of Dental Amalgams. Int. J. of Dentistry. Vol. 2011:1-7.

26. Mutis M, Pinzón J, Castro G. Las amalgamas dentales: ¿un problema de salud pública y ambiental? Revisión de la literatura. Univ. Odontol. 2011 Jul-Dic; 30(65): 63-70. 